\title{
BMJ Open Study protocol for a multicentre, open- label, single-arm phase I/II trial to evaluate the safety and efficacy of ripasudil $0.4 \%$ eye drops for retinopathy of prematurity
}

\author{
Mitsuru Arima (D) , ${ }^{1,2}$ Hirosuke Inoue, ${ }^{3}$ Shintaro Nakao (D) , ${ }^{1,4}$ Akiko Misumi, ${ }^{2}$ \\ Maya Suzuki, ${ }^{2}$ Itsuka Matsushita, ${ }^{5}$ Shunsuke Araki, ${ }^{6}$ Chiemi Yamashiro, ${ }^{7}$ \\ Kazumasa Takahashi, ${ }^{8}$ Masayuki Ochiai, ${ }^{3}$ Noriko Yoshida, ${ }^{9}$ Masayuki Hirose, ${ }^{2}$ \\ Junji Kishimoto, ${ }^{2}$ Koji Todaka, ${ }^{2}$ Shunji Hasegawa, ${ }^{8}$ Kazuhiro Kimura, ${ }^{7}$ \\ Koichi Kusuhara, ${ }^{6}$ Hiroyuki Kondo, ${ }^{5}$ Shouichi Ohga, ${ }^{3}$ Koh-Hei Sonoda ${ }^{1}$
}

To cite: Arima M, Inoue $\mathrm{H}$, Nakao S, et al. Study protocol for a multicentre, open-label, single-arm phase $\mathrm{I} / \mathrm{II}$ trial to evaluate the safety and efficacy of ripasudil $0.4 \%$ eye drops for retinopathy of prematurity. BMJ Open 2021;11:e047003. doi:10.1136/ bmjopen-2020-047003

- Prepublication history for this paper is available online. To view these files, please visit the journal online (http://dx.doi. org/10.1136/bmjopen-2020047003).

Received 17 November 2020 Accepted 13 July 2021

D Check for updates

(c) Author(s) (or their employer(s)) 2021. Re-use permitted under CC BY-NC. No commercial re-use. See rights and permissions. Published by BMJ.

For numbered affiliations see end of article.

\section{Correspondence to}

Dr Mitsuru Arima;

m-arima@eye.med.kyushu-u. ac.jp

\section{ABSTRACT}

Introduction Retinopathy of prematurity (ROP) is a vascular proliferative disorder that occurs in preterm infants. Existing treatments are only indicated in severe ROP cases due to the high invasiveness and the potential risk of irreversible side effects. We previously elucidated that ripasudil, a selective inhibitor of the Rho-associated protein kinase, has the ability to inhibit abnormal retinal neovascularisation in animal models. In addition, ripasudil eye drops (Glanatec ophthalmic solution $0.4 \%$ ) have been already used for the treatment of glaucoma. Since eye drop therapy is less invasive, early intervention for ROP is possible. The purpose of this phase $\mathrm{I} / \mathrm{Il}$ trial is to evaluate the safety and efficacy of ripasudil eye drops for preterm infants with ROP.

Methods and analysis This is a multicentre, open-label, single-arm phase $1 / I$ trial. To evaluate the safety and efficacy of ripasudil as much as possible, ripasudil will be administered to all enrolled preterm infants with zone $\mathrm{l} / \mathrm{ll}$, stage 1, or worse ROP. The safety and efficacy of ripasudil in treated patients will be assessed in comparison to a historical control group. Because this is the first trial of ripasudil in preterm infants, a dose-escalation study (once daily for 1 week, then two times per day for 2 weeks) will be conducted in phase I. After obtaining approval from the independent data and safety monitoring board to continue the trial after the completion of phase I, phase II will be conducted. In phase II, ripasudil eye drops will be administered two times per day for 12 weeks. The primary endpoint in phase II is also safety. Efficacy and pharmacokinetics will be evaluated as secondary endpoints.

Ethics and dissemination This study protocol was approved by the institutional review board at each of the participating centres. Data will be presented at international conferences and published in peer-reviewed journals.

Trial registration numbers NCT04621136 and jRCT2071200047.
Strength and limitations of this study

- Ripasudil has already been used for glaucoma treatment, and no serious adverse events caused by ripasudil eye drops have been reported in adults.

- Treatment with eye drops is less invasive than existing therapies such as laser photocoagulation and intravitreal injection of anti-vascular endothelial growth factor agents.

- Phase II will only be conducted in the absence of safety issues of concern in phase I.

- The limitations of this trial are its open-label and single-arm design, as well as the small number of enrolled patients.

\section{INTRODUCTION}

Retinopathy of prematurity (ROP) is a visionthreatening disease that occurs in preterm infants. Annually, more than 20000 infants worldwide are diagnosed with irreversible visual impairment due to ROP. ${ }^{1}$ Improvements in the management of preterm infants, such as lower oxygen saturation and postnatal nutritional intervention, have led to a decrease in the ROP onset in developed countries. ${ }^{2}$ At the same time, however, improved maternal and neonatal management has also increased the survival rate of extremely preterm infants at high risk of developing severe ROP. ${ }^{3}$ Younger gestational age and lower birth weight exacerbate the severity of ROP ${ }^{4}$ therefore, there is a risk that the incidence of severe ROP may increase in the future.

Preterm infants are vulnerable to stress and easily develop circulatory failure. ${ }^{5-8}$ As the number of treatments for extremely preterm 
infants with ROP is expected to increase, the development of a less invasive treatment option is needed. We have demonstrated that the Rho-associated protein kinase (ROCK) is activated in the mouse retina exposed to oxygen-induced retinopathy (OIR) ${ }^{9}$ a widely used animal model of ROP. ${ }^{10}$ We have confirmed that eye drops of ripasudil, a selective ROCK inhibitor, reduce the area of the ischaemic retina and inhibit abnormal retinal angiogenesis. ${ }^{9}$ We have also shown that ROCK activated by vascular endothelial growth factor (VEGF), which is upregulated in the ROP retina, causes retinal inflammation by promoting the entry of inflammatory cells into the retina and activation of inflammatory cells. ${ }^{911}$ These results indicate that ripasudil eye drops have the potential to inhibit VEGF-induced and cytokine-induced abnormal angiogenesis and promote normal angiogenesis.

Ripasudil eye drops (Glanatec ophthalmic solution $0.4 \%$ ) have already been used for the treatment of glaucoma. ${ }^{12}{ }^{13}$ Ripasudil is rapidly metabolised and, therefore, is less likely to accumulate in the body. Results of pharmacokinetic studies in the plasma of adults have shown that the half-life values of ripasudil and its metabolite M1 (activity is $1 / 6$ th of ripasudil) were 0.5 hour and 2.6 hours, respectively. The long-term safety of ripasudil eye drops in adults has also been examined in several studies and there have been no reports of serious adverse events (SAEs). ${ }^{14-16}$

Eye drop therapy is less invasive compared with the existing treatment options such as laser photocoagulation (LPC) and intravitreal injection of anti-VEGF agents (anti-VEGF therapy). ${ }^{17-19}$ This facilitates early intervention, which is a major advantage of the eye drop therapy. The indication for LPC or anti-VEGF therapy is defined as 'type 1 ROP (stage 2 or 3 in zone II with plus disease, stage 3 in zone I with or without plus disease or stage 1 or 2 disease in zone I with plus disease)' due to concerns about treatment invasiveness, post-treatment complications and recurrence. ${ }^{17}{ }^{19-23}$ If the infant's general condition worsens, it becomes difficult to even perform an ophthalmic examination. Because the examination to decide the indication for an LPC or anti-VEGF treatment cannot be conducted, it is possible that the disease deteriorates to type 1 ROP. By contrast, eye drop therapy can be continued even if the examination cannot be performed and the ROP progression can be prevented. To establish the early therapeutic intervention with ripasudil eye drops, the safety and efficacy of ripasudil in premature infants need to be fully ascertained.

\section{METHODS AND ANALYSIS \\ Study design}

This is a multicentre, open-label, single-arm, investigatorinitiated phase I/II trial to evaluate the safety and efficacy of ripasudil in preterm infants with ROP. Phase I is conducted at Kyushu University Hospital, and phase II is conducted at Kyushu University Hospital, Hospital of the

\section{Box 1 Inclusion and exclusion criteria}

\section{Inclusion criteria}

1. Informed consent signed by parents or legal guardians of the patient.

2. Patients born with $\leq 32$ weeks of gestational age and/or $\leq 1500 \mathrm{~g}$ of birth weight.

3. Patients with the following types of ROP in both eyes:

- Zone I ROP with stage $\geq 1$.*

- Zone II ROP with stage $\geq 1$.*

Exclusion criteria

1. Patients with aggressive posterior ROP in one or both eyes.

2. Patients with type 1 ROP† in one or both eyes.

3. Patients with a confirmed or suspected chromosomal abnormality or genetic disorder.

4. History of hypersensitivity to ripasudil (including drugs with similar chemical structure) in patients or their mothers.

5. Patients or their mothers have participated in another intervention study and have used the study drug of another intervention study within 30 days prior to enrolment in this study or within 5 times the half-life of the study drug.

6. Patients with an ocular structural abnormality that can affect the evaluation of this clinical study by investigators.

7. Patients with a clinically significant neurological disease (eg, intraventricular hemorrhagehaemorrhage grade 3 or higher, periventricular leukomalacia and severe hydrocephalus with significantly increased intracranial pressure).

8. Patients with inadequate blood access.

9. Patients with complications, allergies or worsening systemic conditions that make it difficult to conduct this trial.

10. Patients judged unsuitable by investigators.

$\mathrm{ROP}$, retinopathy of prematurity.

*Zones and stages are defined according to the International Classification of Retinopathy of Prematurity Revisited. ${ }^{24}$

†Type 1 ROP means stage 2 or 3 in zone II with plus disease, stage 3 in zone I with or without plus disease or stage 1 or 2 disease in zone I with plus disease.

University of Occupational and Environmental Health and Yamaguchi University Hospital.

\section{Eligibility criteria}

After obtaining informed consent from patients' parents or legal guardians, potential participants will be screened by investigators. The inclusion and exclusion criteria are shown in box 1 . The zone and stage of ROP are defined according to the International Classification of Retinopathy of Prematurity Revisited. ${ }^{24}$

\section{Endpoints}

The primary endpoint of this study is the safety of ripasudil in preterm infants with ROP. Ophthalmic and systemic examinations, as well as clinical laboratory and physiology tests, will be performed to examine the incidence of local and systemic adverse events (AEs) and adverse drug reactions (ADRs). An AE will be registered even if the abnormality occurs in only one eye. Tolerability of ripasudil will also be assessed. The secondary endpoints are the efficacy and pharmacokinetics of ripasudil. The exploratory endpoints include the number of days until ROP worsens to type 1 ROP, the number of days 


\section{Box 2 Evaluation items in this trial}

Primary endpoint
Proportion of patients with AEs and ADRs.
Secondary endpoints
1. Efficacy:
- Proportion of patients whose ROP worsens to type 1 ROP in one
or both eyes (phase II).
- Proportion of patients with ROP remission in both eyes (phase II).
2. Pharmacokinetics of ripasudil (phases I and II).
Exploratory endpoints (phase II)
1. Number of days until ROP worsens to type 1 ROP* (phase II).
2. Number of days until ROP remission† (phase II).
3. Most advanced stage of ROP within the study period (phase II).
ADR, adverse drug reaction; AE, adverse event; ROP, retinopathy of
prematurity.
*If both eyes reach type 1 ROP, the number of days in the eye that
reached it first is used for the analysis.
†The number of days required for ROP remission in both eyes is used
for the analysis.
†lf the most advanced stage is different between the left and right eye,
the stage of the more advanced eye is used for the analysis.

until ROP remission and the most advanced stage of ROP within the study period. The details of each evaluation item are summarised in box 2 .

\section{Historical control}

In order to collect as much information as possible on the safety and efficacy of ripasudil, all infants will receive the ripasudil eye drop therapy in this trial. The survey result of the medical records of 84 infants who underwent ROP screenings with ophthalmic examinations between April 2017 and November 2019 at the clinical trial institutions will be used as a historical control. Of the 84 patients, 27 (33\%) patients developed type 1 ROP by 12 weeks after the onset of stage 1 ROP and received LPC or anti-VEGF therapy. To assess the efficacy of ripasudil, the results of this study will be compared with those of the historical control.

\section{Investigational drug}

Glanatec ophthalmic solution $0.4 \%$ (Kowa Co., Japan) containing ripasudil hydrochloride hydrate will be used for this trial. Glanatec ophthalmic solution $0.4 \%$ was granted marketing approval for the treatment of glaucoma and ocular hypertension in Japan, Korea, Singapore and Malaysia in 2014, 2019, 2020 and 2020, respectively. The investigational drug and its safety information have been obtained from Kowa Co.

\section{Intervention}

Phase I

The study schedule for infants participating from phase I is shown in table 1. A total of three infants participating from phase I will receive one drop of ripasudil ophthalmic solution in both eyes, once daily for 1 week, followed by one drop in both eyes, two times per day for 2 weeks. At the end of the 3 weeks of treatment for the first patient, a data and safety monitoring board (DSMB) will be held to decide whether the second and third patients can be enrolled into phase I. The DSMB will discuss the safety again at the end of the 3-week eye drop treatment for the third patient to determine if phase II can begin. In addition, the three infants who participate in phase I can continue to receive one drop in both eyes, two times per day for additional 9 weeks (12 weeks in total) if the investigators determine that there are no safety issues with ripasudil.

\section{Phase II}

The study schedule for infants participating from phase II is shown in table 2. A total of 21 patients from three clinical trial institutions will receive one drop of ripasudil ophthalmic solution in both eyes, two times per day for 12 weeks. The safety and efficacy will be assessed at the end of the 12-week treatment period. The efficacy of ripasudil will be evaluated in comparison to the results of the historical control. In addition, another follow-up examination will be performed 4 weeks after the end of the treatment.

\section{$A E$ and $A D R$ reporting}

The investigators will collect and record all AEs that occur in patients during the study and follow-up periods. ADR is defined as: (1) the AE disappears after treatment is discontinued, (2) the $\mathrm{AE}$ recurs after treatment is restarted, (3) no other risk factor can explain the occurrence of this $\mathrm{AE},(4)$ a correlation between the dose and duration of the investigational drug administration and the $\mathrm{AE}$ occurrence can be established and (5) there is no rational basis that other treatments are responsible for the onset of this AE. The investigators will determine the $\mathrm{AE}$ severity according to the International Neonatal Consortium (INC) Neonatal AE Severity Scale V.1.0. ${ }^{25}$ AEs will be defined as SAEs in the following cases: (1) death of the patient, (2) the occurrence of AEs that pose a risk of death, (3) the occurrence of AEs that leave the patient permanently disabled or (4) the occurrence of AEs that require hospitalisation or an extended period of hospitalisation.

\section{Rescue treatment}

When the ROP exacerbates to the indicated stage of existing treatments (ie, type 1 ROP), ripasudil eye drops will be discontinued and the rescue treatment (LPC or anti-VEGF therapy) will be performed.

\section{Dose change and stop criteria}

When it is determined that AEs of concern have occurred due to the investigational drug, the investigator will discontinue the drug administration or switch to oncedaily administrations of ripasudil. Ripasudil administration will be discontinued in the following cases: (1) the investigators decide it is difficult to continue the clinical trial due to worsening of other diseases, (2) the investigators or DSMB decide it is difficult to continue the clinical trial due to AEs that do not disappear with withdrawal 


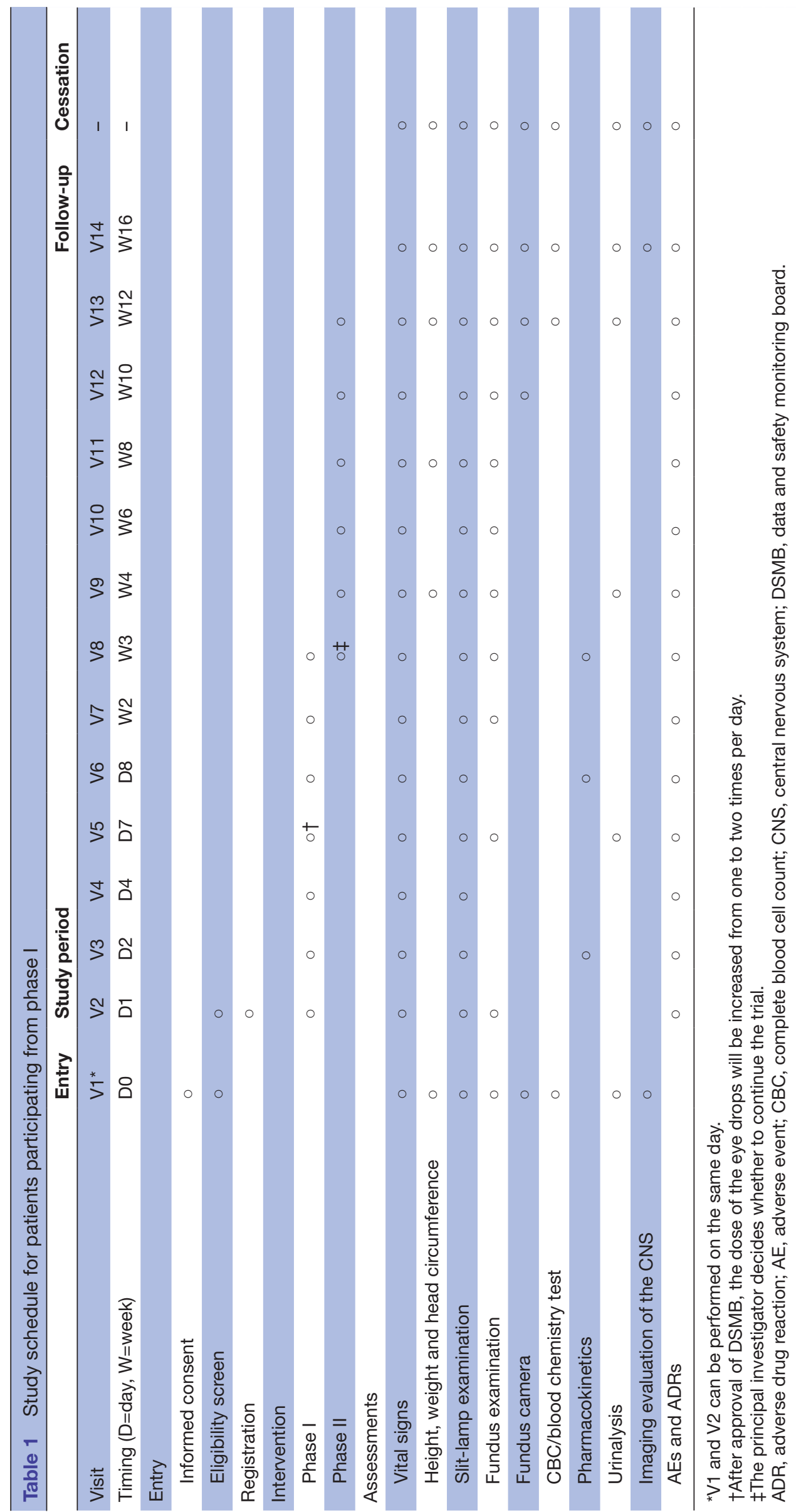

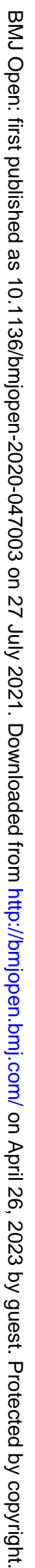


Table 2 Study schedule for patients participating from phase II

Entry Study period $\quad$ Follow-up Cessation

\begin{tabular}{llllllllllllllll}
\hline Visit & V1* & V2 & V3 & V4 & V5 & V6 & V7 & V8 & V9 & V10 & V11 & V12 & V13 & - \\
$\begin{array}{l}\text { Timing (D=day, } \\
\text { W=week) }\end{array}$ & D0 & D1 & D2 & D4 & W1 & W2 & W3 & W4 & W6 & W8 & W10 & W12 & W16 & -
\end{tabular}

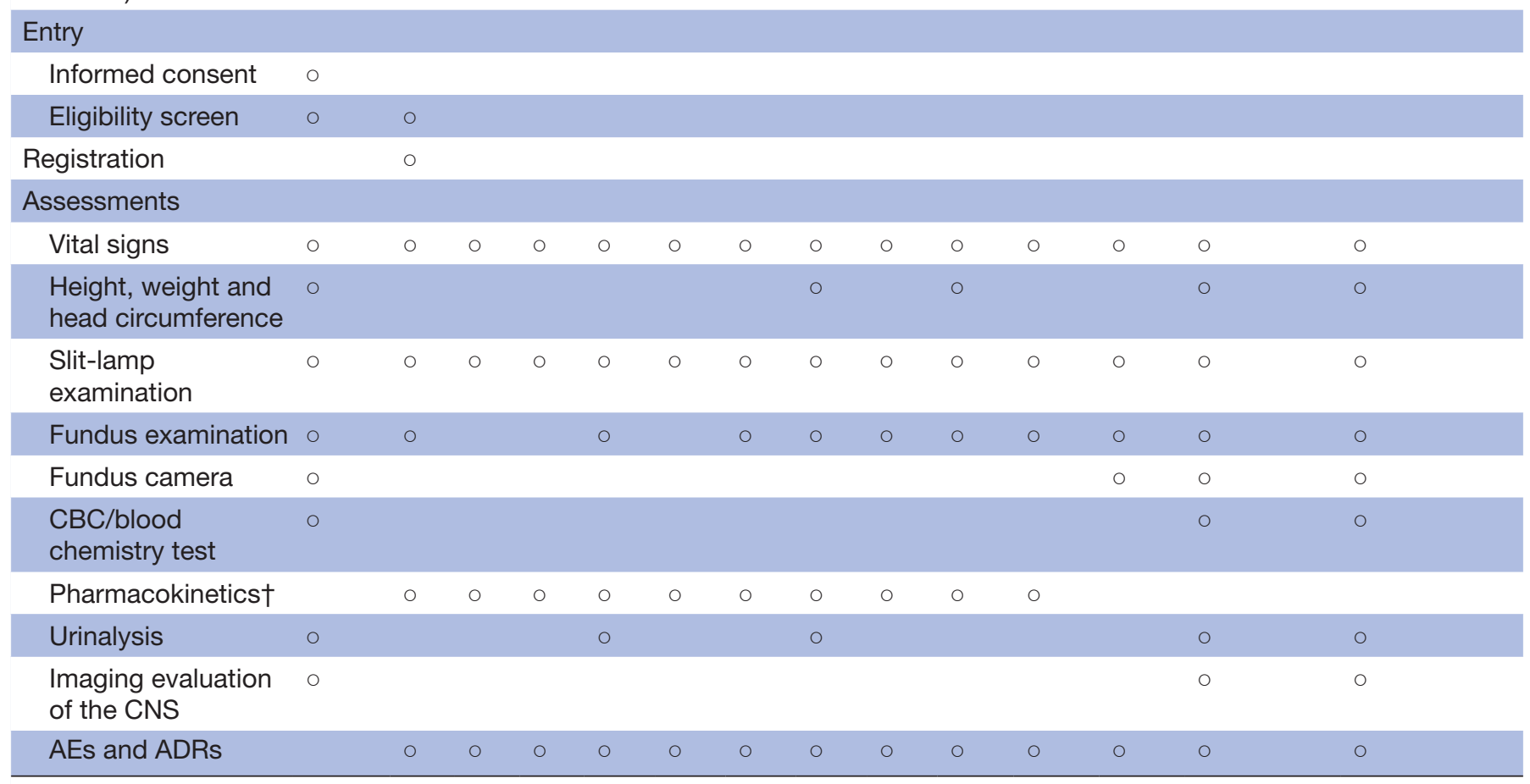

${ }^{*} \mathrm{~V} 1$ and $\mathrm{V} 2$ can be performed on the same day.

†Blood samples for pharmacokinetic studies will be collected 3 times at any point during the study period. Blood will be drawn at any point up to 4 hours after ripasudil administration.

$A D R$, adverse drug reaction; $A E$, adverse event; $C B C$, complete blood cell count; CNS, central nervous system.

or reduction of the dose of the investigational drug, (3) parents or legal guardians of the patients request to discontinue the clinical trial or withdraw the consent and (4) the treatment progress of the patients is impossible to confirm due to the discontinuation of hospital visits or other reasons.

\section{Safety evaluation}

The incidence of AEs and ADRs and tolerability of ripasudil will be evaluated up to 3 weeks in phase I and up to 12 weeks in phase II. Particular attention will be paid to the occurrence of AEs and ADRs that have a significant negative impact on circulatory and respiratory status, neurodevelopment and general condition.

\section{Efficacy evaluation}

In phase II, as shown in box 2, (1) the proportion of patients whose ROP worsens to type 1 ROP in one or both eyes and (2) the proportion of patients with ROP remission in both eyes in ripasudil-treated patients will be compared with the historical control group. The following exploratory endpoints will be also evaluated: (1) number of days until ROP worsens to type $1 \mathrm{ROP}$ in patients receiving the rescue treatment, (2) number of days until ROP remission and (3) the most advanced stage of ROP within the study period in patients presenting ROP remission without treatment. The outcome of each patient at the end of the treatment period (12 weeks after ripasudil administration) will be used for statistical analyses. Fundus photographs taken at study entry and at the end of the treatment period will be confirmed by the independent review committee to assess the validity of the zones and stages determined by the investigators.

\section{Pharmacokinetics}

To examine the concentration of ripasudil and its metabolite M1, blood samples will be collected from patients in phases I and II. In phase I, the minimum plasma concentration (Cmin) at three points of each patient will be investigated to confirm the drug accumulation in the body. If aldehyde oxidase activity (metabolic enzyme of ripasudil) in preterm infants is low, there is a risk of ripasudil accumulation in the body of preterm infants. Therefore, we planned to measure the plasma concentration of ripasudil and M1 at 12 hours and 24 hours after administration. In phase II, population pharmacokinetics will be conducted with sparse sampling of each patient to obtain the profiles of absorption, distribution, metabolism and excretion. As shown in table 1, blood will be collected in 
phase I at visit 3 (24 hours after drug administration), visit 6 (12 hours after drug administration) and visit 8 (at the end of the treatment). In phase II, blood will be collected at any three points from visit 2 to 11 (table 2). The timing can be any time up to 4 hours after administration. Since the patient is a preterm baby, it is difficult to perform multiple blood collections from the same patient at short intervals. Therefore, blood samples will be obtained from multiple patients at different time points. Pharmacokinetic analysis will be performed using non-linear mixed effects modelling. We will estimate the contribution of covariates such as gestational age, postnatal age, postconceptional age and body weight.

For pharmacokinetic analysis, $800 \mu \mathrm{L}$ of blood will be collected from the subject. Peripheral blood samples are obtained by venipuncture. Non-nutritive sucking, facilitated swaddling and holding will be performed for relieving pain during sample collections.

Eye drops cannot be administered properly, especially to babies who are crying or fighting. Therefore, nurses and parents are instructed not to force eye drops into crying babies, but to apply eye drops when they are resting. In the case of failure to apply the drops correctly due to eyelid closure or other reasons, we will allow only one additional application within $30 \mathrm{~min}$. We will keep a record of the time of eye drop application and share this information with pharmacokinetic analysis specialists. After the patients are discharged from the hospital, the parents will be asked to record the eye drop time in a logbook to monitor and confirm the drug administration.

\section{Trial period}

Phase I was conducted between October 2020 and March 2021. Phase II started from April 2021 and will end in July 2022. The registration of patients for phase II will be closed in April 2022.

\section{Sample size}

A total of 24 patients ( 3 in phase I and 21 in phase II) will be enrolled in this trial. Based on the past medical records, the number of new subjects per month who were considered to be eligible for this clinical trial was eight. Assuming a dropout rate of about $10 \%$ for medical record screening, a consent acquisition rate of approximately $35 \%$ and a dropout rate of about $15 \%$ after inclusion, approximately five cases were expected to be included during the planned registration period of phase I. In phase II, the number of newly eligible patients is expected to be approximately 95 and a total of approximately 25 cases can be expected to be included during the registration period.

\section{Statistical analysis}

The following items will be tabulated as demographic and other baseline characteristics: institution, sex, gestational age, postmenstrual age, birth weight, presence of comorbidities, Apgar scores $(1 \mathrm{~min} / 5 \mathrm{~min})$, stage and zone of $\mathrm{ROP}$ and presence of ROP plus disease.
For safety, the number and percentage of cases with AEs will be calculated. We will evaluate the efficacy of ripasudil by comparing the results of the ripasudil-treated and the historical control groups on the following five items:

1. Proportion of patients whose ROP worsens to type 1 ROP in one or both eyes.

2. Proportion of patients with ROP remission in both eyes.

3. Number of days until ROP worsens to type 1 ROP in patients receiving the rescue treatment.

4. Number of days until ROP remission.

5. The most advanced stage of ROP within the study period.

The analysis method for each of these items is shown below:

1. The normal approximation to the binomial distribution will be used to assess whether the proportion of patients who have reached type 1 ROP in the ripasudiltreated group is smaller than the proportion in the historical control group.

2. The normal approximation to the binomial distribution will be used to assess whether the proportion of ripasudil-treated patients with ROP remission is larger than the corresponding proportion in the historical control group.

3. If both eyes reach type 1 ROP, the time in the first eye to reach type $1 \mathrm{ROP}$ is used for the analysis. The Kaplan-Meier method will be used to plot a survival curve and calculate the median, as well as the 25th and 75 th percentile points, for the time to reach type 1 ROP. The two-sided $80 \%$ and two-sided $95 \%$ CIs will be calculated using the method of Brookmeyer and Crowley, respectively. The same analysis will be performed for the historical control group.

4. The time to ROP remission in both eyes will be used in this analysis. The Kaplan-Meier method will be used to create a survival curve and calculate the median, as well as the 25th and 75th percentile points, for the time to reach ROP remission. The two-sided $80 \%$ and two-sided $95 \%$ CIs will be calculated using the method of Brookmeyer and Crowley, respectively. The same analysis will be performed for the historical control group.

5 . The proportions of stages that progressed the most in the study period will be calculated. If the most advanced stages of both eyes are different, the worse will be adopted for this analysis. Then, the percentage of subjects who fall into the following categories will be evaluated to be less than the percentage of the historical control group using the normal approximation method of binomial distribution:

1. Progression from stage 1 to stage 2 or higher (subjects with stage 1 disease in both eyes at baseline).

2. Progression from stage 1 to stage 3 (subjects with stage 1 disease in both eyes at baseline).

3. Progression from stage 2 to stage 3 (subjects with stage 2 disease in the more severely affected eye at baseline). 
In addition, the two-sided $80 \%$ and two-sided $95 \%$ CIs of the relevant number of subjects in the ripasudil eye drop and historical control groups will be calculated using the Wilson method.

\section{Patient and public involvement}

Patients' parents and/or the public were not involved in the design of this study.

\section{ETHICS AND DISSEMINATION}

This trial will be performed according to the ethical principles originating from the Declaration of Helsinki and the Good Clinical Practice guidelines. This trial was approved by the institutional review board of each institution. Informed consent will be obtained from parents or legal guardians of each patient before the registration. All data will be collected by investigators confidentially. We will present the results of this trial in international peer-reviewed journals and at international and national conferences. Approval for this study has been obtained from the IRB of Kyushu University Hospital (Approval No. 2020-767).

\section{DISCUSSION}

The development of a less invasive treatment modality for ROP is urgently needed. The ultimate goal of this study is to expand the indication of Glanatec ophthalmic solution to ROP. This phase I/II will collect the information necessary to conduct phase III. The primary endpoint of this trial is safety. While the safety of ripasudil has been documented in adults, ${ }^{14-16}$ it is not well evaluated in preterm infants. We are concerned about the occurrence of possible ADRs as described below.

Because the circulating blood volume of preterm infants is lower than that of adults, the plasma concentration of ripasudil in preterm infants needs to be evaluated. The concentration of ripasudil will not exceed the safety range that has been identified in non-clinical toxicity tests. Thus, it is unlikely that ripasudil eye drops will cause systemic negative effects in premature infants. However, respiratory depression and hypothermia can occur when the plasma concentration of ripasudil exceeds the safety range assessed in non-clinical studies using rats. We, therefore, should particularly pay attention to changes in vital signs.

In clinical trials conducted prior to approval, several ocular ADRs have been reported in adults. The most common ADR is conjunctival hyperemia $(69.0 \%, 457$ out of 662 patients), which is caused by vasodilation due to ROCK inhibition. ${ }^{26}$ This hyperemia is transient and has been known to appear within 1 hour after instillations and persist for 1-3 hours. ${ }^{27} 28$ The next common ADRs are blepharitis and allergic conjunctivitis. In a trial with two times per day doses over 52 weeks, their incidences were $17.8 \%$ (63 out of 354 ) and $15.3 \%$ (54 out of 354 ), respectively. On the other hand, the incidence rates of these unwanted effects in an 8-week study were $0.3 \%$ (1 out of 308 ) and $0.3 \%$ (1 out of 308), respectively. In a postmarketing survey (mean observation period: $96 \pm 27$ days), the incidences of conjunctival hyperemia, blepharitis and allergic conjunctivitis were $4.0 \%$ (122/3058), $0.8 \%$ (24/3058) and $1.4 \%$ (42/3058), respectively. These results indicate that the incidences of these ADRs are not expected to be high in this trial with its 12-week study period. However, preterm infants secrete less tear fluid than full-term infants, ${ }^{29}$ which may lead to prolonged retention of ripasudil in the conjunctival sac. If so, there is a risk of an increased incidence of ocular ADRs. In adults, blepharitis and allergic conjunctivitis were both reversible and disappeared following treatment discontinuation. Therefore, if the onset of these ADRs is observed in this trial, the dose should be reduced or the drug discontinued immediately.

In a non-clinical study using dogs, lens opacity was observed along the suture line following administration of ripasudil eye drops at a high concentration for 13 weeks. The non-toxic dose in dogs is 10 times the clinical dose, so there is a sufficient safety range. However, the epithelial barrier function is weak in newborns. ${ }^{30}$ Instilled drugs are either absorbed through the corneal or non-corneal route, and ripasudil is considered to be mainly absorbed through the corneal route. ${ }^{31}$ Since the epithelial barrier function of the cornea and conjunctiva is reduced in newborns, the ripasudil concentration in the anterior chamber is possibly elevated. Okumura et al reported that ROCK inhibition promotes the adhesion of corneal endothelial cells, inhibits apoptosis and increases the number of proliferating cells. ${ }^{32}$ A slit-lamp examination will be performed at all visits to exclude the occurrence of corneal endothelial abnormalities as well as a cataract. To examine the effect of ripasudil on ocular development, the fundus photographs will be evaluated by an independent image evaluation committee and the refractive values will be measured at the end of the trial.

The prevalence of ROP is low ${ }^{1}$; thus, the main limitation of this trial is, besides the lack of a placebo group, the small number of enrolled patients. To evaluate the safety and efficacy of ripasudil as much as possible, all patients will receive the investigational drug. The primary endpoint of this trial is safety. We aim to obtain the most information on safety as possible from a small population. If necessary, stratified analysis by gestational age or birth weight will be conducted. Another limitation is that OIR, which was used in the efficacy test of ripasudil, is not a disease model that fully reflects the pathology of human ROP. In this model, exposure to continuous hyperoxia (constant exposure of $75 \%$ O2) for 5 days from postnatal day 7 causes vaso-obliteration and relative hypoxia due to withdrawal from hyperoxia induces vasoproliferation. ${ }^{10}$ In contrast, in humans, oxygen is often administered immediately after birth, and concentrations as high as $75 \%$ are not administered because high oxygen administration is associated with ROP severity and lung damage. Both the American Academy of Pediatrics and European 
consensus guidelines recommend a target $\mathrm{SpO}_{2}$ in the range of $90 \%-95 \%$, and the minimum concentration of oxygen needed to maintain this range is given to preterm infants. ${ }^{334}$ In addition, pathological angiogenesis occurs in OIR, but this disappears spontaneously with time. Therefore, OIR is a disease model that can only reproduce stage 2 of human ROP. Although the efficacy of investigational drugs for ROP is mainly tested using OIR, the results may not be completely extrapolated to human ROP. OIR has been widely used worldwide for ROP studies, but it has these limitations.

If we can confirm the safety and efficacy of ripasudil for ROP in this trial, we will continue to prepare for a phase III study. In parallel with this study, we also plan to conduct an observational study to verify the long-term safety of ripasudil. This study will follow the development of visual function and neurodevelopment in children up to the age of 3 years.

\section{Author affiliations}

${ }^{1}$ Ophthalmology, Graduate School of Medical Sciences, Kyushu University, Fukuoka, Japan

${ }^{2}$ Center for Clinical and Translational Research, Kyushu University Hospital,

Fukuoka, Japan

${ }^{3}$ Pediatrics, Graduate School of Medical Sciences, Kyushu University, Fukuoka, Japan

${ }^{4}$ Ophthalmology, National Kyushu Medical Center, Fukuoka, Japan

${ }^{5}$ Ophthalmology, University of Occupational and Environmental Health, Kitakyushu, Japan

${ }^{6}$ Pediatrics, University of Occupational and Environmental Health, Kitakyushu, Japan ${ }^{7}$ Ophthalmology, Yamaguchi University Graduate School of Medicine, Ube, Japan

${ }^{8}$ Pediatrics, Yamaguchi University Graduate School of Medicine, Ube, Japan

${ }^{9}$ Ophthalmology, Fukuoka Dental College, Fukuoka, Japan

Acknowledgements The authors thank Dr Toshio Hisatomi (Fukuoka University Chikushi Hospital), Dr Tadamune Kinjo (Fukuoka Children's Hospital) and Tetsuya Matoba (Kyushu University) for their contributions as the members of the independent safety evaluation committee and Dr Miwako Goto (Fukuoka Children's Hospital) and Dr Yuji Oshima (Saiseikai Fukuoka General Hospital) for their contributions as the members of the independent image evaluation committee.

Contributors MA is the principal investigator of this trial and wrote the manuscript with critical assistance from KTo, SH, KKi, KKu, HK, SO and K-HS. Authors MA, HI, AM and MS conceived and designed the trial protocol. MA, HI, IM, SA, CY, KTa, $\mathrm{MO}, \mathrm{SH}, \mathrm{KKi}, \mathrm{KKu}, \mathrm{HK}, \mathrm{SO}$ and K-HS collected the clinical data. SN, NY and KTo supported this project from a regulatory perspective. All authors contributed to multiple revisions of the trial protocol and approved the final manuscript version for publication. MH and JK are responsible for the statistical analyses in this study.

Funding This research was supported by the Japan Agency for Medical Research and Development (AMED) under Grant Number JP20ek0109458.

Competing interests SN and Kowa Co., Ltd. (Japan) have disclosed the patent W0 2014/174747

Patient and public involvement Patients and/or the public were not involved in the design, or conduct, or reporting, or dissemination plans of this research.

Patient consent for publication Parental/guardian consent obtained.

Ethics approval Approval for this study has been obtained from the IRB of Kyushu University Hospital (Approval No. 2020-767).

Provenance and peer review Not commissioned; externally peer reviewed.

Open access This is an open access article distributed in accordance with the Creative Commons Attribution Non Commercial (CC BY-NC 4.0) license, which permits others to distribute, remix, adapt, build upon this work non-commercially, and license their derivative works on different terms, provided the original work is properly cited, appropriate credit is given, any changes made indicated, and the use is non-commercial. See: http://creativecommons.org/licenses/by-nc/4.0/.
ORCID iDs

Mitsuru Arima http://orcid.org/0000-0002-6081-8133

Shintaro Nakao http://orcid.org/0000-0003-4200-0052

\section{REFERENCES}

1 Blencowe H, Lawn JE, Vazquez T, et al. Preterm-associated visual impairment and estimates of retinopathy of prematurity at regional and global levels for 2010. Pediatr Res 2013;74 Suppl 1:35-49.

2 Fang JL, Sorita A, Carey WA, et al. Interventions to prevent retinopathy of prematurity: a meta-analysis. Pediatrics 2016;137:e20153387.

3 Stoll BJ, Hansen NI, Bell EF, et al. Trends in care practices, morbidity, and mortality of extremely preterm neonates, 1993-2012. JAMA 2015;314:1039-51

4 Kim SJ, Port AD, Swan R, et al. Retinopathy of prematurity: a review of risk factors and their clinical significance. Surv Ophthalmol 2018;63:618-37.

$5 \mathrm{Ng}$ PC, Wong SPS, Chan IHS, et al. A prospective longitudinal study to estimate the "adjusted cortisol percentile" in preterm infants. Pediatr Res 2011;69:511-6.

6 Washio Y, Uchiyama A, Nakanishi H, et al. Hemodynamic analysis in infants with late-onset circulatory collapse. Pediatr Int 2013;55:582-8.

7 Kawai M. Late-onset circulatory collapse of prematurity. Pediatr Int 2017;59:391-6.

8 Arima M, Tsukamoto S, Fujiwara K, et al. Late-onset circulatory collapse and continuous positive airway pressure are useful predictors of treatment-requiring retinopathy of prematurity: a 9-year retrospective analysis. Sci Rep 2017;7:3904.

9 Yamaguchi M, Nakao S, Arita R, et al. Vascular normalization by ROCK inhibitor: therapeutic potential of ripasudil (K-115) eye drop in retinal angiogenesis and hypoxia. Invest Ophthalmol Vis Sci 2016;57:2264-76.

10 Smith LE, Wesolowski E, McLellan A, et al. Oxygen-induced retinopathy in the mouse. Invest Ophthalmol Vis Sci 1994;35:101-11.

11 Arima M, Nakao S, Yamaguchi M, et al. Claudin-5 redistribution induced by inflammation leads to Anti-VEGF-Resistant diabetic macular edema. Diabetes 2020;69:981-99.

12 Tanna AP, Johnson M. Rho kinase inhibitors as a novel treatment for glaucoma and ocular hypertension. Ophthalmology 2018;125:1741-56.

13 Tanihara $\mathrm{H}$, Inoue $\mathrm{T}$, Yamamoto $\mathrm{T}$, et al. One-year clinical evaluation of $0.4 \%$ ripasudil $(\mathrm{K}-115)$ in patients with open-angle glaucoma and ocular hypertension. Acta Ophthalmol 2016;94:e26-34.

14 Tsukahara S, Enomoto N, Ishida K, et al. One-year efficacy and safety assessment of ripasudil, a Rho kinase inhibitor, in an addition to or replacing existing treatment regimens: a retrospective study. $J$ Ocul Pharmacol Ther 2020;36:512-21.

15 Tanihara H, Kakuda T, Sano T, et al. Safety and efficacy of ripasudil in Japanese patients with glaucoma or ocular hypertension: 3-month interim analysis of ROCK-J, a post-marketing surveillance study. Adv Ther 2019;36:333-43.

16 Maruyama Y, Ikeda Y, Mori K, et al. Safety and efficacy of long-term ripasudil $0.4 \%$ instillation for the reduction of intraocular pressure in Japanese open-angle glaucoma patients. J Ocul Pharmacol Ther 2020;36:229-33.

17 Early Treatment for Retinopathy of Prematurity Cooperative Group, Good WV, Hardy RJ, et al. Final visual acuity results in the early treatment for retinopathy of prematurity study. Arch Ophthalmol 2010;128:663-71.

18 Mintz-Hittner HA, Kennedy KA, Chuang AZ, et al. Efficacy of intravitreal bevacizumab for stage $3+$ retinopathy of prematurity. $N$ Engl J Med 2011;364:603-15.

19 Stahl A, Lepore D, Fielder A, et al. Ranibizumab versus laser therapy for the treatment of very low birthweight infants with retinopathy of prematurity (rainbow): an open-label randomised controlled trial. Lancet 2019;394:1551-9.

20 Houston SK, Wykoff CC, Berrocal AM, et al. Laser treatment for retinopathy of prematurity. Lasers Med Sci 2013;28:683-92.

21 Davitt BV, Quinn GE, Wallace DK, et al. Astigmatism progression in the early treatment for retinopathy of prematurity study to 6 years of age. Ophthalmology 2011;118:2326-9.

22 Quinn GE, Dobson V, Davitt BV, et al. Progression of myopia and high myopia in the early treatment for retinopathy of prematurity study: findings at 4 to 6 years of age. J Aapos 2013;17:124-8.

23 Garcia Gonzalez JM, Snyder L, Blair M, et al. Prophylactic peripheral laser and fluorescein angiography after bevacizumab for retinopathy of prematurity. Retina 2018;38:764-72. 
24 International Committee for the Classification of Retinopathy of Prematurity. The International classification of retinopathy of prematurity revisited. Arch Ophthalmol 2005;123:991-9.

25 Salaets T, Turner MA, Short M, et al. Development of a neonata adverse event severity scale through a Delphi consensus approach. Arch Dis Child 2019;104:1167-73.

26 Kameda T, Inoue T, Inatani M, et al. The effect of Rho-associated protein kinase inhibitor on monkey Schlemm's canal endothelial cells. Invest Ophthalmol Vis Sci 2012;53:3092-103.

27 Tanihara $\mathrm{H}$, Inoue T, Yamamoto $\mathrm{T}$, et al. Phase 1 clinical trials of a selective Rho kinase inhibitor, K-115. JAMA Ophthalmol 2013;131:1288-95.

28 Tanihara $\mathrm{H}$, Inoue T, Yamamoto $\mathrm{T}$, et al. Intra-ocular pressurelowering effects of a Rho kinase inhibitor, ripasudil (K-115), over 24 hours in primary open-angle glaucoma and ocular hypertension: a randomized, open-label, crossover study. Acta Ophthalmol 2015;93:e254-60.
29 Isenberg SJ, Apt L, McCarty J, et al. Development of tearing in preterm and term neonates. Arch Ophthalmol 1998;116:773-6.

30 Kusari A, Han AM, Virgen CA, et al. Evidence-based skin care in preterm infants. Pediatr Dermatol 2019;36:16-23.

31 Isobe T, Kasai T, Kawai H. Ocular penetration and pharmacokinetics of ripasudil following topical administration to rabbits. $\mathrm{J} O c u l$ Pharmacol Ther 2016;32:405-14.

32 Okumura N, Ueno M, Koizumi N, et al. Enhancement on primate corneal endothelial cell survival in vitro by a rock inhibitor. Invest Ophthalmol Vis Sci 2009;50:3680-7.

33 Cummings JJ, Polin RA. Committee on fetus and newborn. oxygen targeting in extremely low birth weight infants. Pediatrics 2016;138:e20161576.

34 Sweet DG, Carnielli V, Greisen G, et al. European Consensus Guidelines on the Management of Respiratory Distress Syndrome 2019 Update. Neonatology 2019;115:432-50. 